\title{
Design and Simulation of a NDIR Gas Sensor System with Ray Tracing Using a Monte-Carlo Method
}

\author{
Johann Mayrwöger ${ }^{1}$, Wolfgang Reichl ${ }^{2}$, Peter Hauer $^{3}$, Christian Krutzler ${ }^{4}$, Bernhard Jakoby ${ }^{1}$ \\ ${ }^{1}$ Institute for Microelectronics and Microsensors, Johannes Kepler University Linz, Linz, Austria \\ ${ }^{2} \mathrm{E}+\mathrm{E}$ Elektronik, Engerwitzdorf, Austria \\ ${ }^{3}$ Institute for Soft Matter Physics, Johannes Kepler University Linz, Linz, Austria \\ ${ }^{4}$ Integrated Microsystems Austria (IMA), Wiener Neustadt, Austria
}

\begin{abstract}
We report on the use of statistic ray tracing to predict the effectiveness of 3D chamber geometries for NDIR-gas sensors. We outline the method and show examples on how to apply the method to a particular 3D-model of a simple NDIR-sensor design.
\end{abstract}

Keywords: infrared, ray tracing, gas detection

\section{Introduction}

NDIR-gas sensors consist of basic building blocks, i.e., an IR-source, an optical path containing the sample gas, a filter, and an IR-detector. Every single part affects the behavior of the whole system. To minimize development time and costs, we investigate an efficient approach to simulate the entire sensorsystem in the beginning of the design process such that the most promising designs can be rapidly identified and pursued in the subsequent prototype engineering phase. For the analysis of optical systems, which are in the order of several thousand wavelengths, the utilization of geometrical optics rather than field-theoretic electromagnetic modeling is indicated. Specifically, we decided to use a ray tracing method applied to our specific problem (fundamentals on ray tracing in general and ray tracing in combination with gas analysis can be found, e.g., in [1],[2],[3]).

For the results discussed below, the sample gas is $\mathrm{CO} 2$ corresponding to a center wavelength of the filters of $4.26 \mu \mathrm{m}$ [4], the IR-sources are micro-lamps or IR-emitters, and the components for the IRdetection are bolometers or thermopiles both with integrated wavelength-dependent filters for the specified gas. The walls of the optical path are considered to be coated with a reflecting layer to act as IR mirrors. (The fabrication of the system is beyond the scope of this paper and will be discussed elsewhere.)

The described modeling approach consists of two steps, the ray tracing computation and the postprocessing of the ray tracing results, which will be outlined in the following.

\subsection{Ray Tracing}

All parts of the analyzed sensor design are entered into a 3D-raytracing model (specifically, we used the program ZEMAX). The software is capable of modeling a defined number of rays leaving the IR-source in random directions. Depending on the optical path (i.e., the design of the sensor cell), the number of rays reaching the IR-detector and their individual path length within the absorbing gas varies. The associated statistics obtained from the ray tracing model contains the numbers of reflections by the walls, the total path length through the gas, and the incidence angle at the detector for every single ray hitting the detector.

\subsection{Processing of the Ray Tracing Results}

The intensity of every single ray reaching the target is influenced by a couple of parameters: 
- The initial intensity is supposed to be contributed like black body radiation at a defined temperature $\mathrm{T}$

- The wavelength depending emissivity of the IR-source material (in our case tungsten) has to be taken into account [5]

- If micro-lamps are used, the transmission through the glass-bulb around the IR-source [6] is important

- Although the walls of the optical path are considered to be IR-mirrors they are not perfect, every reflection decreases the ray intensity. The reflection loss depends on the coating material - gold in our case [7]. In the ray tracing approach, a maximum number of reflections is set, if a particular ray exceeds this number it is considered extinct.

- According to Lambert-Beer law (I...Intensity after a path length $l, c \ldots$ concentration of the absorbing gas, $\alpha$...absorption coefficient)

$$
I=I_{0} \cdot e^{-\alpha \cdot c \cdot l}
$$

not just the concentration of the sample gas but also the length of the path through the absorbing medium influences the intensity decrease, which is the desired sensing effect. This means that the factor "gas concentration times total path length through the gas" is the general basis for the calculation of the gas concentration response of the entire sensor system.

- Due to the reason that the supported wavelength of an interference filter is angle-dependent, it is necessary to take care of the angles between the incident rays and the gas specific interference filter in front of the detector

Assigning proper parameters for each of these items, using the statistics obtained from the ray tracing model, and summing up (integrating) over all wavelengths thus enables to determine the total intensity at the detector.

\section{Optimization of a Given Sensor System}

The previously described method will now be used to optimize a 3D-model of a simple NDIR-sensor design (see Figure 1, Figure 2, Figure 13, and Figure 14). In particular, we want to find out what IRsource (IR-emitter or small light bulb) in combination with what detector (thermopile detector or bolometer) yields the best sensor signal resolution for the given system.

Additionally, we are interested in how the sensor signal depends on the diameter of the circular optical path of the model. For that reason we varied the diameter of the optical path during the simulations from $1 \mathrm{~mm}$ to $6 \mathrm{~mm}$. In the following, sample results for all of these designs are discussed.

\subsection{Gas Sensor Design with a Commercial IR-Emitter}

In this section, the analysis of a NDIR-system with a commercial IR-emitter as IR-source is explored. For the simulation, the size of the diameter of the circular optical path is changed to study the dependence on this parameter. The analysis is performed for two different types of IR-detectors: a thermopile detector (Figure 1) and a bolometer (Figure 2). Both detectors have an active detecting area of about $1.2 * 1.2$ $\mathrm{mm}^{2}$

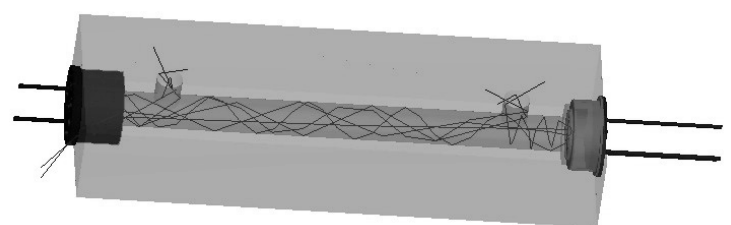

Figure 1: 3D-model of a simulated NDIR-system with an IR-emitter as source (left) and a thermopile sensor as detector (right), cell length $60 \mathrm{~mm}$, diameter of the circular optical path $4 \mathrm{~mm}$.

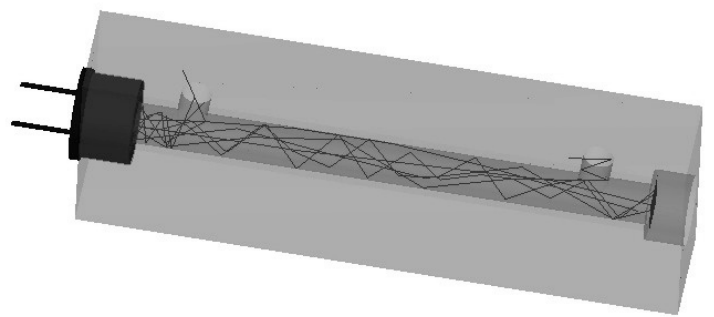

Figure 2: 3D-model of a simulated NDIR-system with an IR-emitter as IR-source (left) and a bolometer as detector (right), cell length $60 \mathrm{~mm}$, diameter of circular optical path $4 \mathrm{~mm}$. 
For this simulation we use $10^{4}$ rays starting at the IR-source, where, for the sake of clarity, just a few of these rays are shown in Figure 1 and Figure 2. The maximum number of considered reflections was set 2000. The chosen temperature of the IR-emitter is 500K.

The two different detectors affect the model by means of the used wavelength-depending filter type:

- Thermopile detector: commercial thermopiles have an integrated filter of high order with sharp edges built in a TO-39 package

- Bolometer: the considered system is based on a $3 \lambda / 4$ interference filter with no housing (specified in [8],[9])

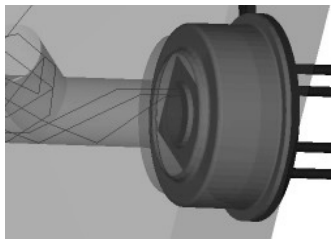

Figure 3: Commercial thermopile in a TO-39 package.

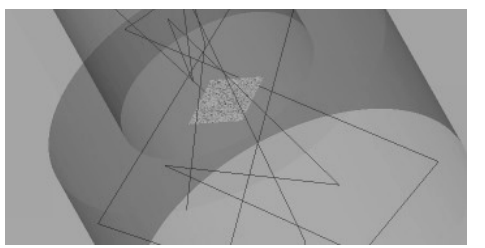

Figure 4: Bolometer with no housing.

The ray tracing model for a particular design provides the following parameters, which have to be analyzed in a post-processing step yielding the overall system response.

- Path lengths for every ray through the absorbing gas (see Figure 5 and Figure 6):

On the basis of Lambert-Beer law (1), a larger average path length through the absorbing gas of the bolometer system improves the sensitivity of the system.

- Number of reflections on the walls for every ray (see Figure 7 and Figure 8):

The tooth like distribution of the associated statistical distribution for this parameter illustrates the fact that the rays starting on the emitter have to "fit" into the given geometry in the sense that only rays with certain emitting angles can reach the detector. Like the previous parameter, this distribution is also affected by the different housing of the two detectors.

- Incidence angle on the filter for every ray (see Figure 9 and Figure 10):

Due to the housing of the thermopile detector, only rays with a small angle can impinge to the filter (the area from $70^{\circ}$ to $80^{\circ}$ in Figure 9 is due to ricochets between the housing and the filter). Because of the missing detector housing, a system with a bolometer does not show this advantage. Thanks to the directional characteristics of the filter and its wavelengthdependence, the thermopile detector yields a better signal resolution.

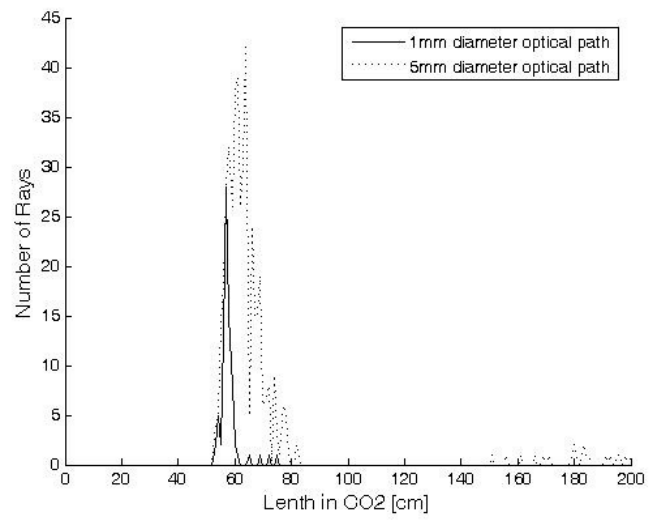

Figure 5: Statistical distribution of the path lengths through the absorbing gas (IR-emitter and thermopile detector, only the incoming rays are considered).

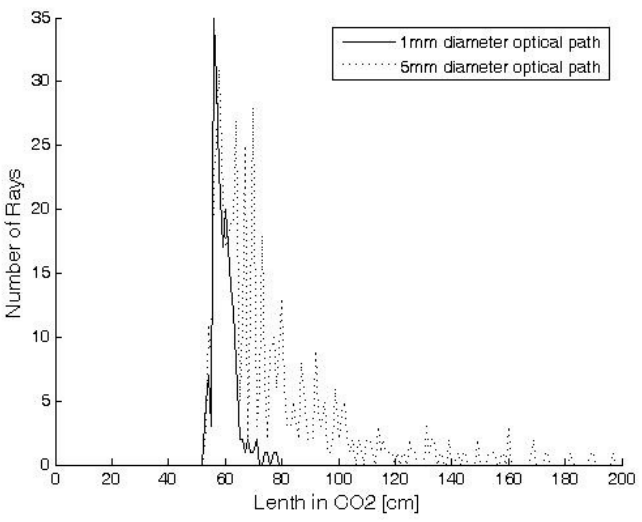

Figure 6: Statistical distribution of the path lengths through the absorbing gas (IR-emitter and bolometer, only the incoming rays are considered). 


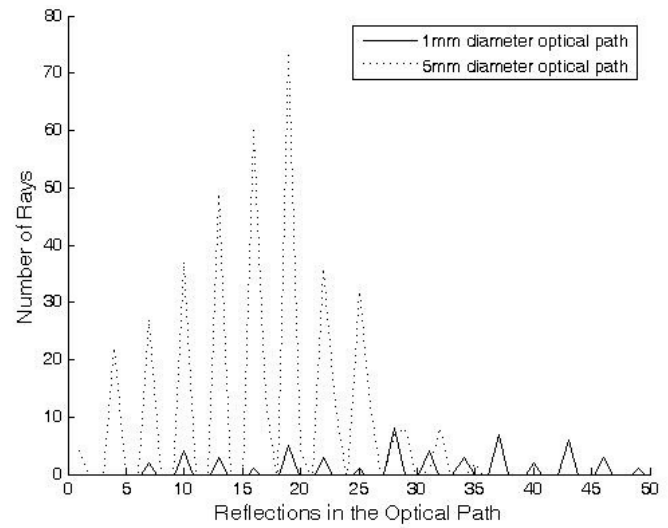

Figure 7: Statistic distribution of the number of reflections on the walls (IR-emitter and thermopile detector, only the incoming rays are considered).

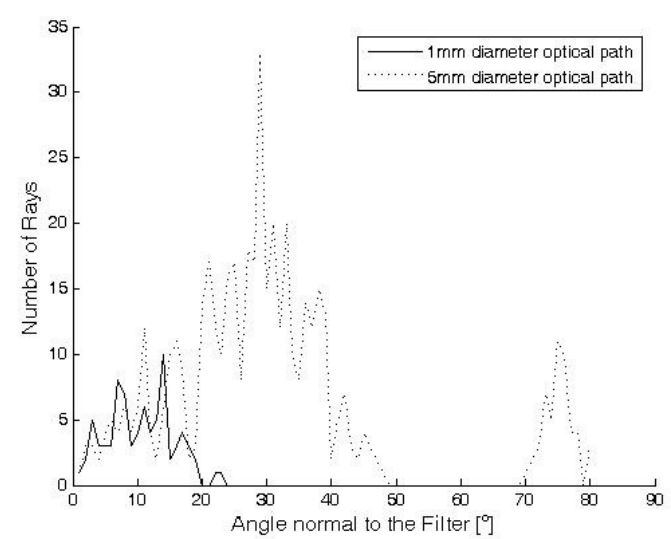

Figure 9: Statistic distribution of the incidence angles (with respect to the surface normal) on the filter (IRemitter and thermopile detector, only the incoming rays are considered).

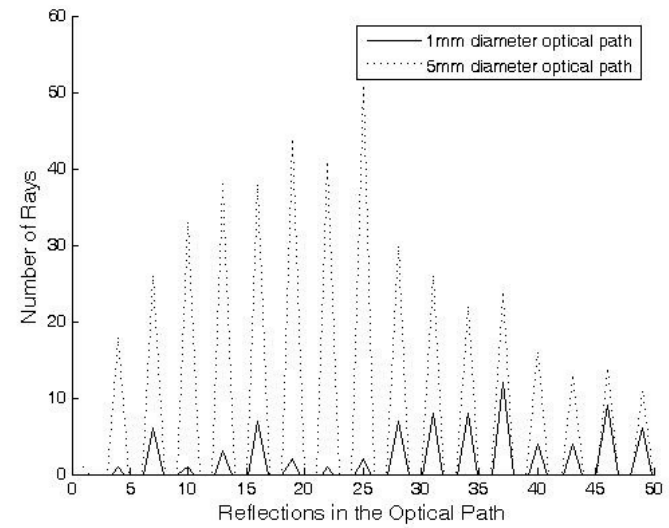

Figure 8: Statistic distribution of the number of reflections on the walls (IR-emitter and bolometer, only the incoming rays are considered).

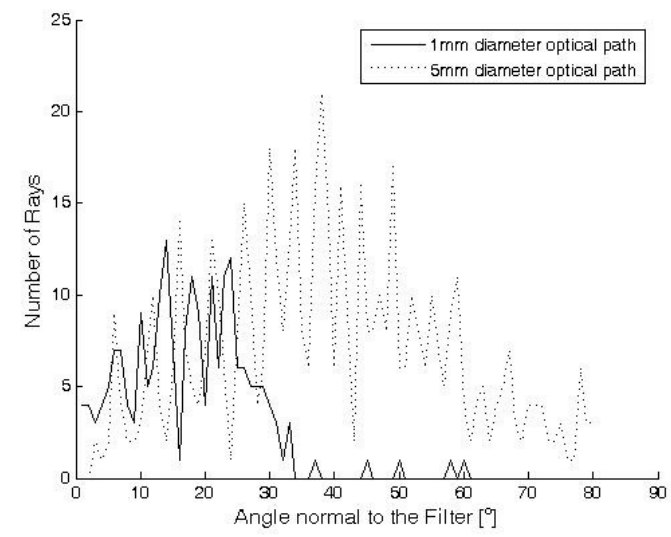

Figure 10: Statistic distribution of the incidence angles (with respect to the surface normal) on the filter (IRemitter and bolometer, only the incoming rays are considered).

Figure 11 and Figure 12 show the results after post processing of the ray tracing data with the corresponding parameters discussed above, i.e. the dependence of the detector signal on the $\mathrm{CO}_{2}$ concentration. In particular, a reflectivity of $99 \%$ at the chamber walls has been assumed. From the results it can be seen that if a thermopile detector is used, the optimum value for the diameter of the circular optical path for the chosen design would be about $5 \mathrm{~mm}$. For the case of a bolometer, no such optimum could be found. Generally it was found that for the particularly studied geometry and the use of a commercial IR-emitter as IR-source, a thermopile detector yields a much better performance than a bolometer.

The reasons are the narrow-band-characteristics of the interference filter of the thermopile detector (compared to the $3 \mathrm{~N} / 4$ filter of the bolometer), and the housing, which blocks unwanted rays with large incidence angles (with respect to the surface normal) on the filter. 


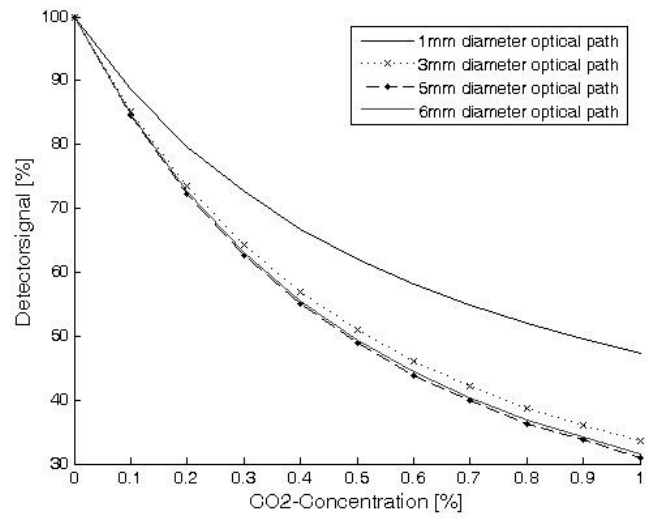

Figure 11: Ray intensity at the detector vs. gas concentration (IR-emitter and thermopile detector).

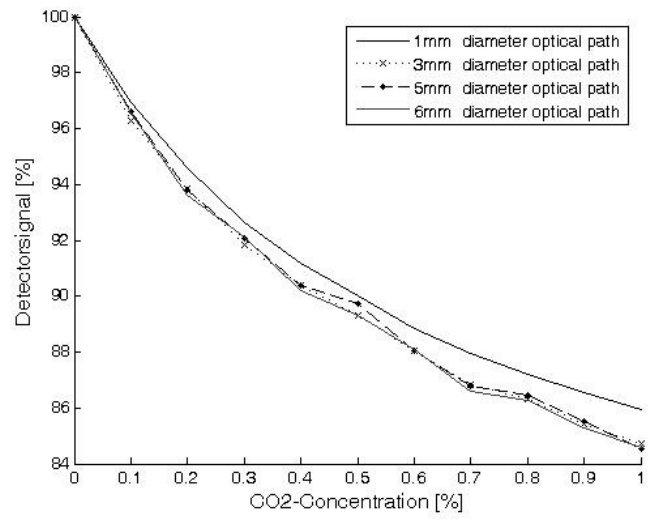

Figure 12: Ray intensity at the detector vs. gas concentration (IR-emitter and bolometer, note the different axis scaling compared to Figure 11).

\subsection{Gas Sensor Design with a Light Bulb as IR-Source}

Compared to the previous section, we now consider an ordinary light bulb as IR-emitter (see Figure 13, Figure 14) featuring a filament temperature of about $1800 \mathrm{~K}$. We considered $10^{5}$ starting rays (instead of $10^{4}$ ). Again we compare the performance for the two different detector types. The ray tracing analysis yields similar results as shown above and is thus not discussed any further.

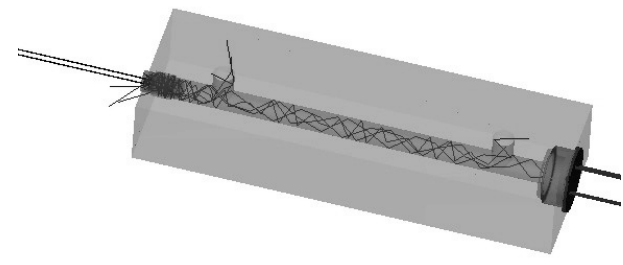

Figure 13: 3D-model of a simulated NDIR-system with a light bulb as source (left) and a thermopile sensor as detector (right), cell length $60 \mathrm{~mm}$, diameter of the circular optical path 3mm.

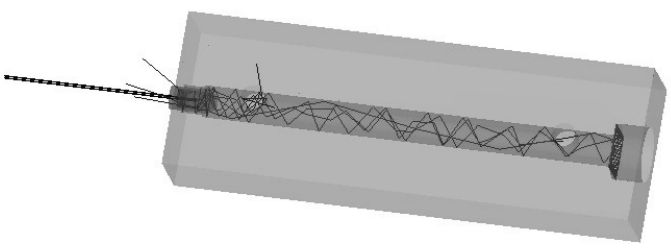

Figure 14: 3D-model of a simulated NDIR-system with a light bulb as IR-source (left) and a bolometer as detector (right), cell length $60 \mathrm{~mm}$, diameter of circular optical path $4 \mathrm{~mm}$.

For the post processing, the same parameters as above have been used, the results are shown in Figure 15 and Figure 16. Utilizing a bolometer in this design yields almost no sensitivity to the gas concentration (note the axis scaling). The major causes are the higher temperature of the IR-source in combination with the filter characteristics of the $3 \mathrm{~N} / 4$ filter associated with the bolometer.

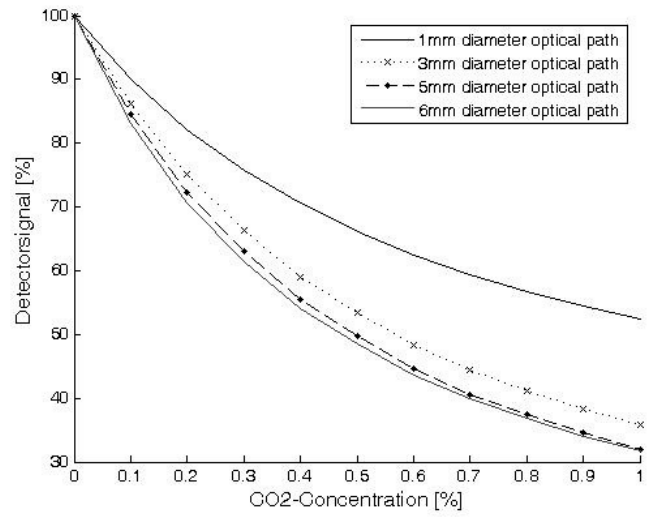

Figure 15: Ray intensity at the detector vs. gas concentration (light bulb as IR-emitter and thermopile detector).

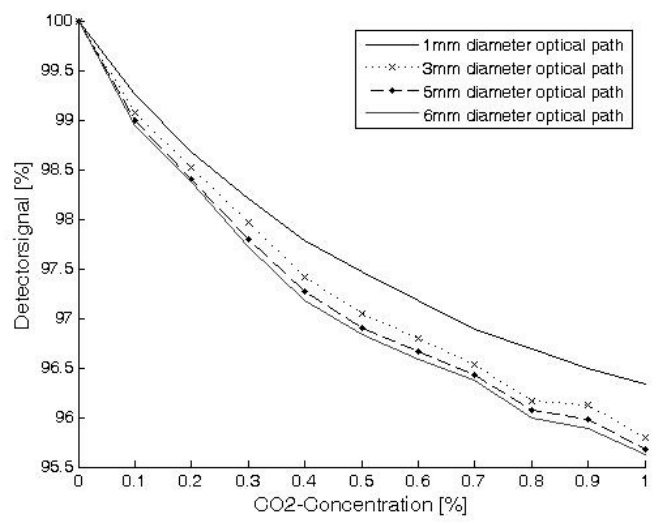

Figure 16: Ray intensity at the detector vs. gas concentration (light bulb as IR-emitter and bolometer). 


\subsection{Comparison of the Considered Designs}

Using a thermopile detector, both IR-sources considered yield virtually identical performance and in both cases the achieved signal resolution is much better than that obtained with the considered bolometer detector.

An interesting result from the ray-tracing simulations is that for both considered IR-sources, only a small fraction of the starting rays are reaching the detector (see Figure 17 and Figure 18). In particular, the calculations show that geometries with larger diameters of the circular optical path do not necessarily yield a maximum number of rays hitting the detector. At the same time, this parameter (the number of surviving rays) is not a direct indicator for the final signal resolution. In any case, the dependence on the diameter of the circular optical path is not a crucial design parameter as it was expected initially.

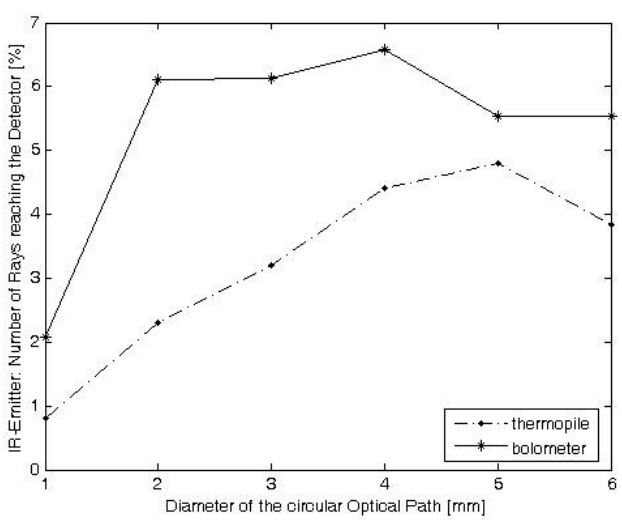

Figure 17: Number of rays reaching the detector for a commercial IR-emitter.

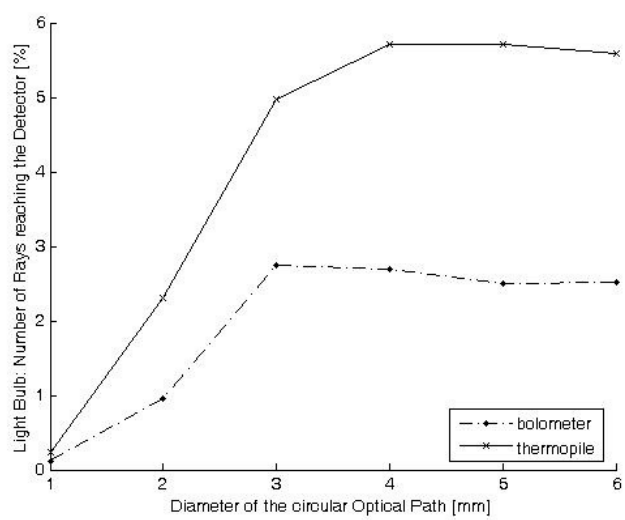

Figure 18: Number of rays reaching the detector for a light bulb as IR-emitter.

\section{Summary and Conclusions}

We demonstrated the use of ray tracing for the analysis of the performance of NDIR-gas sensor systems. As an example, some particular designs have been analyzed and discussed. We considered different types of emitters and detectors and the influence of the characteristics associated with these components on the overall system performance. Using this method, every change in the design including its components can be evaluated very easily such that time and prototyping costs can be minimized during the development.

\section{Acknowledgements}

This work is supported by the Federal Ministry of Economics and Labour and the Federal Government of Lower Austria (Industrial Center of Competence program, project KOFIRS 03301).

[1] Glassner Andrew S., "An introduction to ray tracing", Academic Press, London, 1989

[2] K. Keränena, M. Blomberg, J. Tenhunena and P. Kariojac, "Analytic and raytrace modeling of a miniaturized infrared spectrometer module", MSM 2000, San Diego, p. 660-663

[3] Sieber, I., Eggert, H., Suphan, K.-H., Bechtold, S., "Simulation and Modeling of a Chamber of Analysis of an IR-Gas Sensor", MICRO SYSTEM Technologies 2001, p. 71-76

[4] HITRAN database, www.hitran.com

[5] Touloukian, Yerman S., "Thermophysical Properties of Matter", Vol. 7 - Thermal Radioactive Properties, IFI/Plenum, New York, 1970

[6] www.heraeus-quarzglas.de

[7] David R. Lide, "Handbook of Chemistry and Physics", 86th Edition 2005-2006

[8] Johannes Preiner, Andreas Springer, „Wellenlängenselektive IR-Emitter und Absorber für die Gasanalyse“, Linz, 2005

[9] Jürgen Kasberger, Patrick Rauter, Bernhard Jakoby, „Wavelength Selectivity of a Thermal IR-Absorber as Part of a Fully Integrated IR-Absorption Sensor", IEEE Sensors 2008, Lecce, p. 996-999 